\title{
Ist Sport wirklich eine effektive Therapie?
}

Fragestellung: Die vorliegende Metaanalyse ging der Frage nach, wie effektiv Sport und Bewegung bei dieser Patientengruppe (Alter $>60$ Jahre) eingesetzt werden können.

Hintergrund: Depressionen stellen die häufigste psychische Erkrankung im höheren Lebensalter dar und sind mit erhöhter körperlicher Morbidität und Mortalität verbunden.

Bridle C, Spanjers K, Patel S et al. Effect of exercise on depression severity in older people: systematic review and meta-analysis of randomised controlled trials. Brit J Psychiatry 2012; 201: $180-5$
Patienten und Methodik: Aufgrund der hohen qualitativen Anforderungen konnten insgesamt nur neun Studien 667 Probanden $69 \%$ weiblich, Durchschnittsalter je nach Studie zwischen 65 und 80 Jahren) eingeschlossen werden, davon gingen sieben in die Metaanalyse ein. In der Regel wurde ein gemischtes Training durchgeführt, das sowohl Kraft- als auch Ausdauerkomponenten enthielt. Typischerweise gab es drei bis fünf Trainingseinheiten pro Woche (30-45 Minuten) über einen Zeitraum von drei bis vier Monaten.

Ergebnisse: Es ergab sich eine Assoziation zwischen sporttherapeutischer Interventionen und signifikant niedrigeren Depressionswerten. Dies war unabhängig davon, ob die Diagnose in den Studien klinisch oder über eine Symptom-Checkliste gestellt wurde.

Schlussfolgerungen: Die Ergebnisse zeigen, dass auch bei älteren Menschen mit klinisch relevanten depressiven Symptomen ein den individuellen Fähigkeiten angepasstes therapeutisches Sportprogramm zu einer Abnahme der depressiven Symptomatik führt.

\section{- Kommentar von Andreas Broocks, Schwerin}

\section{Die Herausforderung ist, ein geeignetes Trainingsprogramm zu finden}

Es handelt sich um eine methodisch hochwertige Metaanalyse mit strengen Auswahlkriterien: Es wurden nur randomiserte, kontrollierte Studien eingeschlossen, in denen eine klinisch valide Methode zur Quantifizierung der depressiven Symptomatik, ein strukturiertes und begleitetes Trainingsprogramm sowie eine Studiendauer von mindestens drei Monaten verwendet wurden. Von 2.933 ursprünglich gefundenen Artikeln wurde daher am Ende nur neun Studien eingeschlossen. Bei der Interpretation der Ergebnisse sollten die folgenden Limitationen beachtet werden:

1. Ein grundsätzliches Problem solcher Metaanalysen besteht darin, dass Studien mit sehr unterschiedlichem Design zusammengefasst werden müssen. So war es in einer der berücksichtigten Studien erforderlich, dass die Patienten auf eine antidepressive Medikation eingestellt waren, während dies bei drei anderen Studien ein Ausschlusskriterium war.

2. Es muss weiter berücksichtigt werden, dass aufgrund der Einschlusskriterien nur eine Subgruppe der depressiv erkrankten älteren Patienten für die sporttherapeutische Intervention infrage kam, nämlich Patienten, bei denen keine somatischen Kontraindikationen und keine kognitiven Einschränkungen vorlagen. Wie hoch der Prozentsatz älterer depressiver Patienten ist, bei denen ein körperliches Trainingsprogramm von ausreichender Intensität möglicherweise gar nicht durchführbar ist, bleibt unklar.

3. Auch die Frage der Adhärenz kann nicht beantwortet werden. Nur in einer der Studien wurde ermittelt, dass zwölf Monate nach Beginn der Intervention immerhin noch 57\% der Teilnehmer regelmäßige sportliche Aktivitäten angaben.

4. Die vorgenommene Auswertung erlaubt keine Antwort auf die Frage, ob sich die sportinduzierten therapeutischen Ef- fekte bei Betroffenen mit einer gering ausgeprägten depressiven Symptomatik von den Wirkungen bei schwerer depressiv erkrankten Personen unterscheiden.

5. Ein grundlegendes Problem besteht darin, dass Studien mit negativen Ergebnissen häufig nicht publiziert werden und daher auch nicht in Metaanalysen berücksichtigt werden.

Fazit: Die vorliegende Untersuchung unterscheidet sich von früheren Reviews dadurch, dass konsequenterweise keine nicht randomisierten Studien berücksichtigt wurden. Die berechnete Effektstärke der sporttherapeutischen Intervention (standardized mean difference $=\mathrm{SMD}=0,34$, Konfidenzintervall $0,17-0,52$ ) liegt in derselben Größenordnung wie die Effektstärken für antidepressiv wirkende Medikamente (SMD = 0,2-0,5) oder psychotherapeutische Verfahren (SMD =0,18$0,34)$. Die große klinisch-therapeutische Herausforderung liegt darin, für den individuellen Patienten mit einer depressiven Erkrankung ein tägliches Bewegungsprogramm zu finden und umzusetzen, das die sehr unterschiedlichen körperlichen Beeinträchtigungen dieser Patientengruppe berücksichtigt.

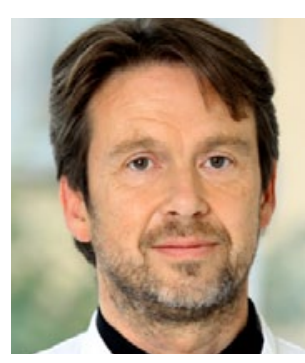

Prof. Dr. med. Andreas Broocks, Schwerin

Ärztlicher Direktor, Klinik für Psychiatrie und Psychotherapie, HELIOS Kliniken Schwerin E-Mail: andreas.broocks@ helios-kliniken.de 